\title{
Solid intracystic papillary carcinoma in male breast: case report
}

\author{
Vanessa Geron ${ }^{1}$, Evelyn Borges Queiroz ${ }^{1}$, Fernanda Santos Lopes ${ }^{1} \mathbb{C}^{\circ}$, \\ Elisabete Lilian Dair ${ }^{1}$ [으, Marcelo Ballaben Carloni ${ }^{1}$ (1)
}

\section{ABSTRACT}

The intracystic papillary carcinoma (IPC) is one of the rarest types of breast cancer, mainly in men, representing less than $1 \%$ of the malignant diseases in the male sex. It is frequently associated with the ductal carcinoma in situ (DCIS), but there are also other forms such as the pure and the invasive ones. The male population breast cancer diagnosis is late and, therefore, it has a worse prognosis. The diagnosis is given by imaging tests and anatomopathological studies. The treatment consists of excisional tumor therapy, which can be carried out conservatively or through mastectomy, with or without adjuvant therapy.

KEYWORDS: breast neoplasms, male; carcinoma, papillary; carcinoma, intraductal, noninfiltrating.

\section{INTRODUCTION}

Breast cancer in men is rare, accounting for about $1 \%$ of all breast cancers and associated with less than $0.1 \%$ mortality. Its incidence, as well as in women, is also related to age, as it mainly affects men aged over 60 years. The five-year overall and event-free survival is low, mainly due to the late diagnosis. When this diagnosis is made, a neoplasm in a more advanced stage is identified ${ }^{1,2}$.

Intracystic papillary carcinoma (IPC) is among the rarest forms of breast cancer, with an incidence of less than $1 \%$ of breast neoplasms. It is usually verified in older women, but it also affects men, though more uncommonly. It has a good prognosis. Its 10 -year survival rate is $100 \%$ and the recurrence-free rate is $95 \%$, which shows that, despite being a rare cancer, it has a high survival rate and a low recurrence rate ${ }^{1-8}$. Thus, to document the occurrence of breast cancer in men is deemed very relevant to identify possible risk factors, to develop more specific therapeutic strategies and even future prevention measures.

Therefore, this study aimed to report the case of a male patient diagnosed with breast cancer, as well as his clinical history and the histological subtype of the tumor, in addition to analyzing the therapeutic approach and its follow-up.

\section{CASE REPORT}

FRS, man, 41 years old, identified the presence of a painless nodule in the left breast in the retroareolar region and sought medical care in
December 2018. On that occasion, ultrasound and bilateral screening mammogram were performed, which showed, respectively, a 1.3 $\mathrm{cm}$ nodulation in the left breast, well-delimited, in the retroareolar, hypoechoic and Bi-rads III region, and a well-delimited nodulation in the central region of the left breast of $1.2 \mathrm{~cm}$ and Bi-rads 0 .

Five months after undergoing these tests, the patient sought new medical care in May 2019. During this consultation, bulging in the left areolar region was identified on the physical examination, on static inspection, and its accentuation, on dynamic inspection. On palpation of the breast, a hardened nodulation of approximately $2 \mathrm{~cm}$ in diameter was observed in the retroareolar region, irregular and adhered to adjacent planes. In the armpits, bilateral fibroelastic lymph nodes were detected, and breast expression was negative.

After clinical evaluation, a new ultrasound was requested and a nodular growth of $0.26 \mathrm{~cm}$ was observed, with a new diameter of $1.56 \mathrm{~cm}$. Furthermore, irregular contours and Bi-rads IVa were found, which demonstrated significant tumor growth in the last five months. An excisional biopsy was chosen for anatomopathological study due to the location and superficiality of the nodulation.

Postoperatively, the patient developed seroma and a small area of necrosis in the areolar region (Figure 1).

The anatomopathological report of the excision of the breast nodule showed an epithelial proliferativelesion with an extensive area of tumor necrosis. The residual neoplasm sample showed cells with mild atypia arranged in solid and cribriform arrangements. Mitotic figures were not observed and the surgical margins were compromised(Figure 2A). 
To confirm the diagnosis, the specimen was referred to immunohistochemical study, which showed encapsulated papillary carcinoma, ${ }^{9}$ with an intermediate-grade ductal carcinoma in situ component in the adjacent parenchyma (Figure 2B). Histological sections demonstrated an extensively necrotic papillary lesion, well-delimited, consisting of fibrovascular axes covered by monotonous epithelial cells with atypia.

In the periphery of the lesion, areas of intermediate-grade solid and cribriform ductal carcinoma in situ were identified. No frankly-invasive carcinoma foci were identified in the sample. The presence of associated ductal carcinoma in situ poses greater risk of local recurrence.

The neoplasm was negative for calponin (SMMHC) and p63 protein, confirming the absence of these cells in the papillary stems and in the periphery of the lesion, negative for high molecular weight cytokeratin (CK-14), and showed strong and diffuse positivity for estrogen receptor (ER) (Figure 2B, Table 1).

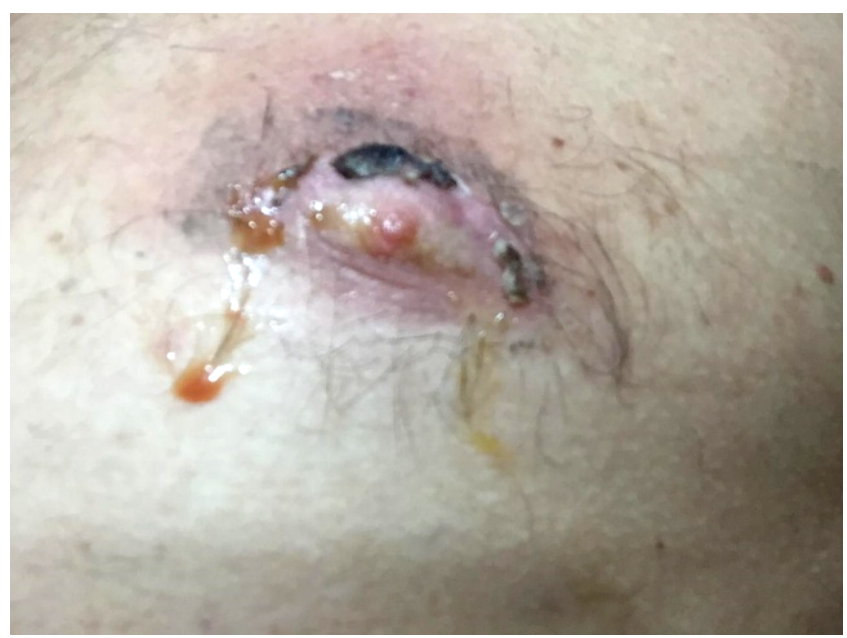

Figure 1. Patient's left breast showing postoperative changes.

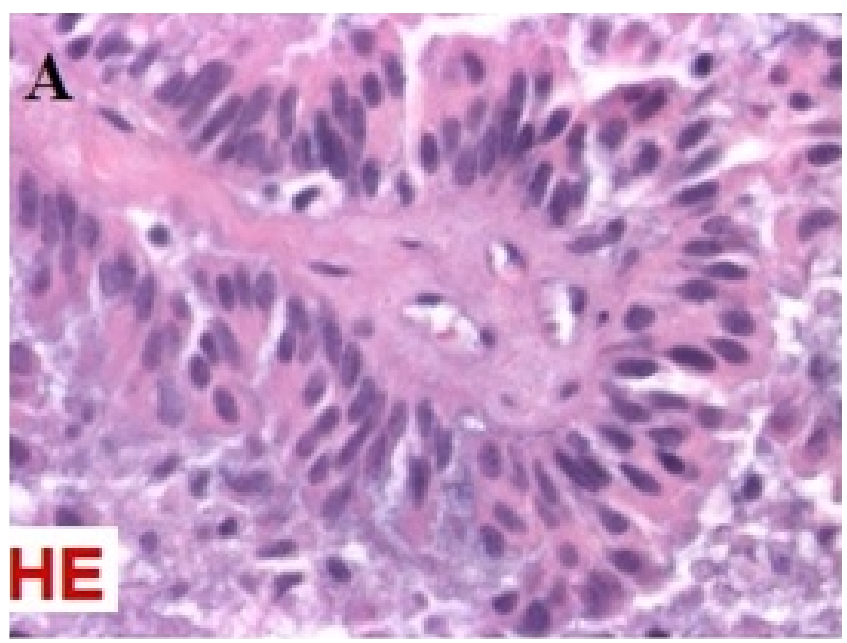

The anatomopathological report also demonstrated an epithelial proliferative lesion with extensive tumor necrosis. The residual neoplasm sample showed cells with mild atypia arranged in solid and cribriform arrangements.

After diagnostic confirmation, the patient underwent simple mastectomy, with isotopic labeling of sentinel lymph node, and had good postoperative evolution. According to the anatomopathological study, a sentinel lymph node free of neoplastic infiltrate and the left breast without residual neoplasia were verified.

Clinical oncology evaluation was requested and Tamoxifen $20 \mathrm{mg} /$ day was prescribed. The patient remained in clinical follow-up after surgery. He showed no signs of local recurrence and has been under outpatient follow-up since the time of diagnosis, in December 2018, with a total follow-up time of three years.

\section{DISCUSSION}

Intracystic papillary carcinoma (IPC) is a rare cancer, representing $1 \%$ of all types of cancer. It accounts for up to $2 \%$ of cases in women, whereas in men the incidence is less than $1 \%$. The average age of its onset ranges from 68 to 84 years, and it mainly affects women, being unusual for men. ${ }^{4}$. It is characterized as intracystic papillary growth carcinoma of the breast, mostly unilateral growth. The diagnosis is usually localized, without dissemination to lymph nodes or with distant metastases ${ }^{3,4,10}$. In the reported patient, the tumor presentation was at 41 years of age, lower than the epidemiological data, and it was well-localized and without dissemination and/or metastases.

Anatomically, this tumor usually appears macroscopically as a well-defined lesion surrounded by a fibrous capsule. Microscopically, the capsule can be filled by a fibrovascular layer, and its stroma is characterized by cells distributed in clearly papillary structures. However, they can present a malignant cellular aspect, with the presence of atypia ${ }^{4,5,10}$.

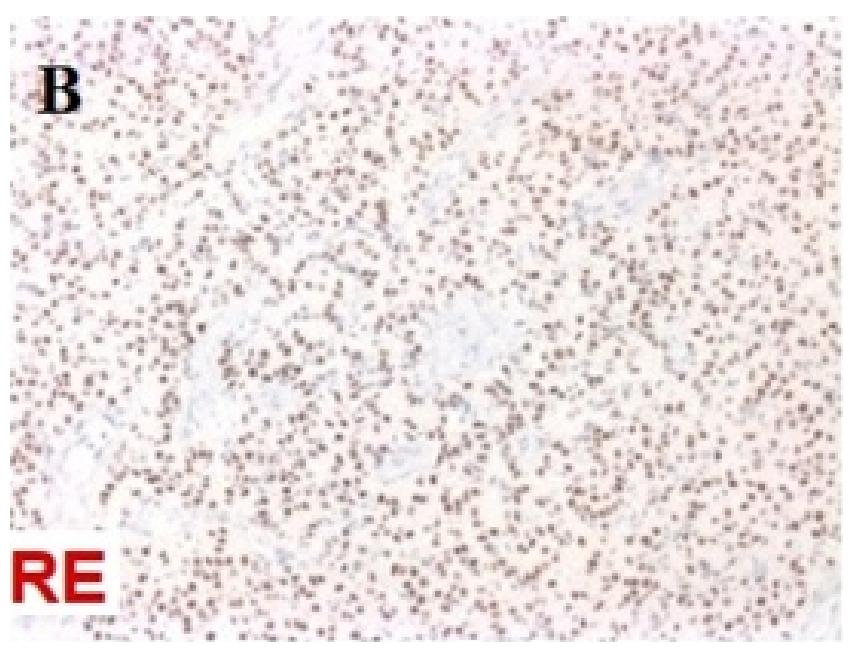

H\&E: hematoxylin and eosin staining; ER: estrogen receptors.

Figure 2. (A) Results of the histopathological study that showed, in the periphery of the lesion, areas of intermediate-grade ductal carcinoma in situ, solid and cribriform types; (B) Immunohistochemical study showing strong and diffuse positivity for estrogen receptors. 
Histologically, IPC is divided into three subtypes: pure, IPC associated with ductal carcinoma in situ (DCIS), and that associated with invasive cancer ${ }^{4,5,10}$. IPC in its pure form is extremely rare. The most frequent form of presentation is associated with DCIS or invasive cancer. In order to differentiate the histological type, studies claim that core biopsy has proved to be effective in differentiating papillary neoplasms from other diseases and from their benign forms ${ }^{6}$. Addressing the nature of the carcinoma is extremely important for the prognosis, as IPC associated with DCIS becomes an important causal factor for the development of invasive carcinoma, requiring additional treatment ${ }^{6}$.

Thus, IPC associated with DCIS refers to a more diffuse form of the disease, which involves several ducts, thus making the lesion more extensive and less localized, favoring the development of invasive carcinoma in addition to posing greater risk of local recurrence ${ }^{3}$.

The progression of carcinoma to the invasive form can be identified by immunohistochemical study, as the high degree of cell differentiation favors the metastatic process of the tumor. The lack of a basal layer in myoepithelial cells (SMMHC) can be identified by calponin and p63, proteins present in myoepithelial cells that, when expressed, indicate that the carcinoma is not yet invasive, i.e., that it is in situ $u^{3,11-13}$. Hence, it is observed that the loss of the basal layer in the myoepithelial cells, i.e., the loss of expression of calponin and p63, assists in the tumor metastatic dissemination, making the carcinoma invasive. Nevertheless, the lack of expression of these receptors increases the chances of this tumor to be malignant. The identification, by immunohistochemistry, of calponin and p63 proteins is highly sensitive in detecting tumor invasion in malignant papillary breast lesions, being widely used in clinical practice ${ }^{3,11-13}$.

Clinical, radiological and immunohistochemical findings are essential for diagnosis. Ultrasonography shows a hypoechoic area with soft tissue echoes projecting from the cyst wall and evidencing an intracystic tumor? ${ }^{7}$. Mammography in IPC is less specific for small tumors and usually becomes inconclusive. Conversely, larger lesions can be described as dense and well-circumscribed masses. Excisional biopsy can be performed on cystic breast lesions, and the anatomopathological study associated with immunohistochemistry helps to make a definitive IPC diagnosis ${ }^{3,13}$.

The differential diagnosis of intracystic papillary lesions is given by histopathological samples and immunohistological studies. Therefore, some authors have reported that differentiation of intracystic papillary carcinoma is also related to loss of heterozygosity $(\mathrm{LOH})$ on chromosome 16q. This characteristic has become a useful marker to differentiate an intracystic papillary carcinoma from an intraductal papillary carcinoma, as it does not have $\mathrm{LOH}^{7.8}$. Thus, by polymerase chain reaction, it is possible to determine the malignant potential of IPC more clearly. The etiology of the lesions is paramount to verify the disease prognosis and, therefore, to analyze an additional treatment plan when feasible ${ }^{6-8}$.

According to the literature, the detection of ER and progesterone increases the probability that the tumor will develop in a favorable way. This is because about $90 \%$ of IPC that are positive for these markers are classified as neoplasms with good prognosis ${ }^{4}$. Furthermore, the presence of $\mathrm{LOH}$ on chromosome 16q in IPC demonstrates that this tumor has a low probability of malignancy, which is an important prognostic factor. However, the negativity expressed by calponin and p63 proteins in myoepithelial cells indicates that this tumor has a greater chance of progressing to an invasive carcinoma, favoring distant metastases. Therefore, despite presenting a worse prognostic factor due to the negativity of the expression of proteins in myoepithelial cells, other factors, such as the expression of ER and the presence of LOH on chromosome 16q, cooperate for the carcinoma of the patient in question to present a good prognosis over time $\mathrm{e}^{11-13}$.

Treatment, according to some studies, should be based on the associated pathology, and there are still no definitive guidelines for treatment. Surgical excision with a safety margin for resection has become the mainstay of treatment and can be conservative or not; in the later case, a mastectomy is required ${ }^{4-16}$.

Regarding the use of hormone replacement therapy, it is not recommended as a routine procedure, considering that there are no changes related to future prognosis. Nevertheless, concerning IPC associated with DCIS or microinvasive disease, patients may receive Tamoxifen therapy due to increased rates of tumor recurrence and the development of invasive carcinoma. Thus, additional treatment is needed to reduce tumor recurrence rates ${ }^{3,16,17}$.

\section{CONCLUSION}

Intracystic papillary carcinoma is an extremely rare cancer, especially in men. Someimmunohistochemical characteristics make this tumor associated with carcinoma in situ to have a better prognosis

Table 1. Result of the immunohistochemical study of the collected sample with positivity only for estrogen and androgen receptors. The remaining was negative.

\begin{tabular}{l|c|c|c}
\hline Antibodies & Clone & Result & Note / Block (\%) \\
\hline Estrogen receptor & ER1 & Positive & 100; $+++/+++(A 2884 / 19)$ \\
\hline Cytokeratin 14 & LL002 & Negative & (A2884/19) \\
\hline Myoepithelial cells (SMMHC) & SMMS-1 & Negative, Myoep. cells & (A2884/19) \\
\hline p63 protein (squamous/transitional epithelia; myoepithelial cells) & DAK-p63 & Negative, Myoep. cells & (A2884/19) \\
\hline Androgen receptor & F39.4.1 & Positive & (A2884/19) \\
\hline
\end{tabular}


such as the presence of ER. Diagnostic investigation is carried out through clinical examination associated with imaging tests, which may be requested during the evaluation. In addition, anatomopathological and immunohistochemical studies can contribute to a better characterization of the carcinoma. The mainstay of initial treatment is surgical excision of the tumor, followed by systemic adjuvant therapy, using Tamoxifen, a selective ER modulator. Therefore, this drug is the most suitable for tumors that express positivity for ER and progesterone. As for radiotherapy, it has been shown to be more effective for IPC associated with DCIS, but it is more suitable for more aggressive cases associated with lymphovascular invasion.

\section{AUTHORS' CONTRIBUTIONS}

V.G.: Conceptualization, data curation, formal analysis, funding acquisition, investigation, methodology, investigation, project administration, supervision, validation, visualization, writing original draft, writing - review \& editing.

E.B.Q.: Conceptualization, data curation, formal analysis, funding acquisition, investigation, methodology, investigation, project administration, supervision, validation, visualization, writing - original draft, writing — review \& editing.

F.S.L.: Conceptualization, data curation, formal analysis, funding acquisition, investigation, methodology, investigation, project administration, supervision, validation, visualization, writing - original draft, writing - review \& editing.

E.L.D.: Conceptualization, funding acquisition, investigation, methodology, investigation, project administration, supervision, validation, visualization, writing - review \& editing.

M.B.C.: Conceptualization, funding acquisition, investigation, methodology, investigation, project administration, supervision, validation, visualization, writing — review \& editing.

\section{REFERENCES}

1. Salomon MFB, Mendonça JV, Pasqualette HAP, Pereira PMS, Sondermman VRM, Manoel VR. Câncer de mama no homem. Rev Bras Mastologia. 2015;25(4):141-5. https://doi.org/10.5327/ Z201500040005RBM

2. Yetkin G, Celayir MF, Tanik C, Citgez B, Uludag M, Mihmanli M. Male breast cancer: A 10 year retrospective case series in a tertiary care hospital. J Pak Med Assoc. 2019;69(8):1209-12.

3. Romics L Jr., O'Brien ME, Relihan N, O'Connell F, Redmond HP. Intracystic papillary carcinoma in a male as a rare presentation of breast cancer: a case report and literature review. J Med Case Rep. 2009;3:13. https://doi.org/10.1186/1752-1947-3-13

4. Luo H, Meng K, He J, Hu Z, Yang O, Lan T, et al. Intracystic papillary carcinoma of the breast in males. Three case reports. Medicine. 2020;99(25):e20278. https://dx.doi.org/10.1097\%2F MD.0000000000020278

5. Barcelos MRB, Vereno Filho AL, Chambô Filho A, Guimarães RA, Cintra LC. Carcinoma Papilífero Intracístico de Mama: Revisão de Literatura e Relato de Dois Casos. Rev Bras Cancerol. 1999;45(3):57-63.

6. Brahmi SA, El M'rabet FZ, Akesbi Y, Benbrahim Z, El Hind AF, Znati K, et al. Intracystic papillary carcinoma associated with ductal carcinoma in situ in a male breast: a case report. Cases J. 2009;2:7260. https://dx.doi.org/10.4076\%2F1757-1626-2-7260

7. SinhaS,HughesRG,RyleyNG.Papillarycarcinomainamalebreast cyst: a diagnostic challenge. Ann R Coll Surg Engl.2006;88(5):W3W5. https://dx.doi.org/10.1308\%2F147870806X129232

8. Kumar M, Pottipati B, Arakeri SU, Javalgi AP. Infiltrating Ductal Carcinoma Co-Existing with Intraductal Papillary Carcinoma of Male Breast: A Rare Case Report. J Clin Diag Res. 2017;11(6):ED04ED05.https://dx.doi.org/10.7860\%2FJCDR\%2F2017\%2F26818.10002

9. Lakhani SR, Ellis IO, Schnitt SJ, Tan PH, Van de Vijver MJ. WHO Classification of Tumours of the Breast. $4^{\mathrm{a}}$ ed. Lyon: IARC; 2012.
10. Ingle SB, Murdeshwar HG, Siddiqui S. Papillary carcinoma of breast: Minireview. World J Clin Cases. 2016;4(1):20-4. https:// dx.doi.org/10.12998\%2Fwjcc.v4.i1.20

11. Russell TD, Jindal S, Agunbiade S, Gao D, Troxell M, Borges VF, et al. Myoepithelial cell differentiation markers in ductal carcinoma in situ progression. Am J Pathol. 2015;185(11):307689. https://doi.org/10.1016/j.ajpath.2015.07.004

12. Li X, Pan B, Song X, Li N, Zhao D, Li M, et al. Breast cancer organoids from a patient with giant papillary carcinoma as a high-fidelity model. Cancer Cell Int. 2020;20:86. https://doi. org/10.1186/s12935-020-01171-5

13. $\mathrm{Hu} \mathrm{ZI}$, Liu $\mathrm{C}$, Fisher PR, Cohen JA. Intracystic papillary carcinoma of the breast in a male patient. Rare Tumors. 2016;8(1):6050. https://dx.doi.org/10.4081\%2Frt.2016.6050

14. Hariprasad S, Hariprasad P, Srinivas T. Intracystic Papillary Carcinoma of the Breast in Males: A Case Report and Review of the Literature. J Clin Diag Res. 2013;7(3):568-70. https://dx.doi. org/10.7860\%2FJCDR\%2F2013\%2F4998.2828

15. Dhebri AR, Ahmad A, Shah N, Arora PK. Intracystic papillary carcinoma of breast: report of three cases and review of the literature. BMJ Case Rep. 2012;2012:bcr2012007237. http:// dx.doi.org/10.1136/bcr-2012-007237

16. Esposito E, Bonito M, Iodice G, Avino F, Donzelli I, Fucito A, et al. Intracystic papillary breast carcinoma with DCIS in a man: a case report. Transl Cancer Res. 2019;8(Suppl. 5):S445-8. http://dx.doi.org/10.21037/tcr.2019.09.40

17. Helland T, Henne N, Bifulco E, Naume B, Borgen E, Kristensen VN, et al. Serum concentrations of active tamoxifen metabolites predict long-term survival in adjuvantly treated breast cancer patients. Breast Cancer Res. 2017;19(1):125. https://doi.org/10.1186/s13058-017-0916-4 